\title{
Ensuring healthy and safe birth: putting women at the center of antenatal care
}

Antenatal care (ANC) can be defined as the care provided by skilled health-care professionals to pregnant women and adolescent girls to ensure the best health conditions for both mother and baby during pregnancy. The components of ANC include risk identification, prevention and management of pregnancyrelated or concurrent diseases, and health education and promotion [1]. Therefore, the purpose of ANC is to maximize a good outcome of delivery, i.e., a healthy baby and no complications in the mother [1]. Therefore, it is necessary to correctly estimate gestational age and to early identify mothers with the potential risk of morbidity and mortality. In addition, several antenatal visits are encouraged to enable ongoing screening of maternal and fetal health status to identify potential problems early on to prevent and minimize potential morbidity. During prenatal visits, mothers and their health-care providers should participate in health education and promotion activities to be undertaken by mothers, family members, and health-care providers as appropriate [1].

Prenatal care can start in the first trimester (ideally within 10 weeks of gestation) for the screening of baseline blood pressure, body weight, and existing chronic health conditions. Referrals to specialists may be needed for those with specific health conditions such as rheumatic heart diseases prevalent in developing countries, complications during the current pregnancy, women with complications in past pregnancies, and women with abnormal physical examination and laboratory results [2]. During the second trimester (preferably before 20 weeks of gestation), efforts should be taken to identify women with abnormal health statuses such as high blood sugar, hypertension, and edema and those with abnormal fetal growth and intrauterine movements. Therefore, ultrasound imaging can be useful to confirm the gestational age and estimate the timing of labor so that preparation can be instituted in case preterm or post-term labor is suspected [3]. Ultrasound imaging can also identify fetal abnormalities and multiple pregnancies such as twins. In a sophisticated healthcare system, genetic studies and counseling can be offered to make informed choices. During the third trimester, certain symptoms warrant attention by the health-care system. These include vaginal bleeding, leakage of fluid per vagina, decreased fetal activity, possible preterm labor such as increased uterine activities and contractions, and high blood pressure. A good referral system is required to avoid obstetrics near misses and reduce birth complications [4]. During the antenatal visits, care should be taken to ensure the safe use of medicine and pain management to minimize the risk of adverse fetal and pregnancy outcomes [5]. Delivery should be carried out by qualified facilities. Screening and referral for depression and anxiety before and after delivery should also be performed [6]. As in any patient group, signs and symptoms suggestive of any medical or surgical conditions should receive due attention and timely referral. Finally, appropriate management should be implemented in the case of stillbirth [7].

From the above descriptions, to receive appropriate care, access to health-care services involves individual and system issues. Timeliness of access to quality care is of paramount importance to handle emergency and urgency situations. Individuals can access quality services when they are geographically within reach and when the available services are financially affordable (such as through insurance coverage). Also, the providers and facilities must be technically competent to handle emergencies. Finally, the services must be trustworthy and acceptable by the patients and their families. The study by Khan and Mehmood in this issue examines the factors associated with utilization of maternal health care using data obtained from the UNICEF's Multiple Indicator Cluster Survey (MIC) conducted by the Bureau of Statistics Punjab, Bureau of Statistics Sindh, Government of the Punjab, and Government of Sindh in Pakistan [8]. They have used the work

*Correspondence to: Editorial Office of Asian Biomedicine, Faculty of Medicine, Chulalongkorn University, Bangkok 10330, Thailand, e-mail: abmjournal@chula.ac.th

O Open Access. ๑ 2020 Editorial Office of Asian Biomedicine, published by Sciendo. (๔) BY-NC-ND This work is licensed under the Creative Commons Attribution NonCommercial-NoDerivatives 4.0 License. 
Behavioral Model of Health Services Utilization by Andersen and Newman for the selection of covariates. Their conclusion that interventions to improve maternal care services should address individual and system variables. Individual variables adversely associated with antenatal visits include maternal education and age, while women with more advanced aged husbands had higher rates of utilization of antenatal services. Women experiencing domestic violence and abuse were less likely to have adequate ANC. System issues encompass the residence in rural areas, family income, and access to media. Women in rural areas had poorer access to health care, while those with higher income and higher access to media are associated with higher rates of antenatal visits. Characteristics that fall under demographics are quite difficult to change; however, more attention and focus can be given to modifiable variables such as improved quality of care and referral systems particularly in rural areas to ensure that safe pregnancy outcome is desirable. Also, patient education and easier access to media can be encouraged. Finally, individual issues such as domestic violence and social issues such as the autonomy of women for control of their lives can also be addressed. Governments and society must work together to cope with both individual and system issues to bring about safe pregnancy and ensure that newborn babies will have the best start of their lives.

\section{References}

[1] World Health Organization Recommendations on Antenatal Care for a Positive Pregnancy Experience. [online] 2016. [cited 2020 Jan 5]. Available from: https://apps.who.int/iris/bitstream/ handle/10665/250796/9789241549912-eng.pdf;jsessionid=03C5275EF8 AD1A2CCC7DE5BD03CD879B? sequence $=1$.

[2] Edwards L, Hui L. First and second trimester screening for fetal structural anomalies. Semin Fetal Neonatal Med. 2018; 23:102-11.

[3] Richter L, Slemming W, Norris SA, Stein A, Poston L, Pasupathy D. Health pregnancy, healthy baby: testing the added benefits of pregnancy ultrasound scan for child development in a randomized control trial. Trials. 2020; 21:25

[4] Abha S, Chandrashekhar S, Sonal D. Maternal near miss: a valuable contribution in maternal care. J Obstet Gynaecol India. 2016; 66(Suppl 1):217-22.

[5] Black E, Khor KE, Kennedy D, Chutatape A, Sharma S, Vancaillie T, Demirkol A. Medication use and pain management in pregnancy: a critical review. Pain Pract. 2019; 19:875-99.

[6] Ayano G, Tesfaw G, Shumet S. Prevalence and determinants of antenatal depression in Ethiopia: A systematic review and meta-analysis. PLoS One. 2019; 14:e0211764.

[7] Bakhbakhi D, Burden C, Storey C, Siassakos D. Care following stillbirth in high-resource settings: Latest evidence, guidelines, and best practice points. Semin Fetal Neonatal Med. 2017; 22:161-6.

[8] Aslam M, Sadiq M, Mehmood T. Assessment of maternal health services utilization in Pakistan: the role of socio-demographic characteristics. Asian Biomed (Res Rev News). 2020; 14:3-7. 